Current Research Topics

in Tropical Diseases; Towards

Successful Control and Elimination

Juntendo Medical Journal

2015. 61 (4), 358-359

\title{
Current Research Topics in Tropical Diseases; Towards Successful Control and Elimination
}

\author{
TOSHIHIRO MITA* \\ * Department of Molecular and Cellular Parasitology, Juntendo University Faculty of Medicine, Tokyo, Japan
}

\section{Introduction}

The extensive spread of infection with Ebola virus disease (EVD) and the resulting death toll in West Africa since 2014 remains a global health priority ${ }^{1)}$. An autochthonous outbreak of dengue fever, that had been recognized as an imported infectious disease ${ }^{2)}$ for the past 70 years or so, simultaneously occurred in Japan. However, a German visitor to Japan developed dengue fever after returning home one year before the 2014 outbreak $^{3)}$. Most tropical diseases except malaria are included in a group called neglected tropical diseases (NTDs) (http: //www. who.int/neglected_ diseases/diseases/en/) that mainly target socially vulnerable groups such as those living in poverty and those residing in remote areas ${ }^{4)}$. Chagas disease, which causes chronic heart failure after a prolonged silent phase, is mostly distributed in remote areas of South and Central America. However, it is also prevalent in the USA with an estimated 300, 000 patients, most of whom are socially vulnerable ${ }^{5)}$. Indeed, opportunities to acquire tropical diseases are increasing because of factors such as global warming and an increase in intercontinental travel among humans and perhaps causative vectors ${ }^{6)}$.

Elimination is defined as a reduction in the incidence of infection in a defined geographical area to zero. Once elimination is achieved, the next step is eradication, namely, permanent reduction of the worldwide incidence to zero ${ }^{7)}$. Only smallpox among infectious diseases has been eradicated so far. The Guinea worm (dracontiasis), filariasis and polio have been eliminated in many endemic regions and these pathogens are approaching eradication. Although 97 countries are still at risk of indigenous infection, malaria has not been reported in 12 countries for 13 years ${ }^{7)}$ and it is gradually approaching elimination in many Southeast Asian countries. The Asia Pacific Leaders Malaria Alliance has set the final goal of eliminating malaria from the Asia-Pacific region by 2030 .

The World Health Organization (WHO) in 1955, embarked on the Global Malaria Eradication Program that was the first attempt to tackle a worldwide infectious disease. However, the campaign relied heavily on a single strategy, namely radical vector control using dichloro-diphenyl-trichloroethane $(\mathrm{DDT})^{8)}$. At that time, vector control alone was thought sufficient to eradicate malaria, despite the fact that the first DDT-resistant mosquito had been identified four years earlier in $1951^{9)}$. In the meantime, all knowledge required to eradicate malaria was already available, but the wide experience and knowledge of malaria investigators were viewed as redundant ${ }^{8)}$. The program therefore precluded further attempts to develop new operational tools and any possibility of novel approaches to attain a deeper understanding of malaria biology, parasitology and epidemiology ${ }^{8)}$. The campaign was discontinued in 1969 without achieving its goal. Although malaria has eliminated in previous endemic regions mostly in

Toshihiro Mita

Department of Molecular and Cellular Parasitology, Juntendo University Faculty of Medicine

2-1-1 Hongo, Bunkyo-ku,Tokyo 113-8421, Japan

TEL: + 81-3-5802-1042 E-mail: tmita@juntendo.ac.jp

[Received Sep. 9, 2015] 
Europe and the USA, it has resurged in other areas. The annual number of patients with malaria in Sri Lanka had dropped to only 17, but the this number surged to 500,000 after the DDT program was stopped ${ }^{10)}$. A comprehensive review of the history of the Global Malaria Eradication Program provides hints for a global fight against tropical diseases. The common perception today is that new technology and further understanding of tropical diseases are absolutely imperative to achieve control and elimination.

This research topic offers a comprehensive review of recent advances in tropical diseases including malaria, filariasis, Chagas disease, EVD and dengue fever. The first article about malaria by Dr. Palacpac and Professor Horii reviews preclinical studies of BK-SE36, a promising blood-stage malaria vaccine that they developed. The malaria parasite possesses numerous ingenious mechanisms to evade human immunity, rendering an effective vaccine difficult to develop. Dr. Hirai focuses on malarial drug resistance, an ongoing obstacle against effective malaria control, and has developed a novel genetic technology. This is discussed with an emphasis on future applications in an operational context, which will enables forecasts about antimalarial resistance. Dr. Kimura reviews the ongoing elimination of lymphatic filariasis under the Global Program to Eliminate Lymphatic Filariasis (GPELF) launched in 2000. This achievement, along with public health and scientific implications are discussed along with basic and translational research and application to field operations. Drs. Nara and Miura summarize the current epidemiology of Chagas disease in non-endemic countries including Japan. This NTD is extant even in developed countries, where it is also neglected. Dr. Yamamoto draws a comprehensive picture of EVD from a basic understanding of the causative pathogen to the recent development of a vaccine and anti-Ebola agents. Dr. Kurosu reviews current advances in understanding dengue fever, with focus on details of the clinical presentation and a putative mechanism of the severe form of this disease.

Tropical medicine research was an important avenue to prevent a reduction of military forces caused by infection with tropical diseases during the age of imperialism. We now believe that tropical diseases are not an indigenous health problem confined to tropical regions; rather, they are global health issues that every country needs to address through collaboration. This special topic will offer further understanding of how academia contributes to global health problems induced by tropical diseases.

\section{References}

1) WHO: Ebola Response Team: Ebola virus disease in West Africa - the first 9 months of the epidemic and forward projections. N Engl J Med, 2014; 371: 14811495.

2) Arima Y, Matsui T, Shimada T, et al: Ongoing local transmission of dengue in Japan, August to September 2014. Western Pac Surveill Response J, 2014; 5: 27-29.

3) Schmidt-Chanasit J, Emmerich P, Tappe D, et al: Autochthonous dengue virus infection in Japan imported into Germany, September 2013. Eurosurveillance, 2014; 19.

4) Hotez PJ: Forgotten People, Forgotten Diseases: The Neglected Tropical Diseases And Their Impact On Global Health And Development. Second Edition: Washington, DC: ASM Press, 2013.

5) Bern C, Kjos S, Yabsley MJ, Montgomery SP: Trypanosoma cruzi and Chagas' disease in the United States. Clin Microbiol Rev, 2011; 24: 655-681.

6) Khasnis AA, Nettleman MD: Global warming and infectious disease. Arch Med Res, 2005; 36: 689-696.

7) WHO: World Malaria Report 2014. Geneva: WHO, 2014.

8) Najera JA, Gonzalez-Silva M, Alonso PL: Some lessons for the future from the Global Malaria Eradication Programme (1955-1969). PLoS Med, 2011; 8: el000412.

9) Livadas G, Georgopoulos G: Development of resistance to DDT by Anopheles sacharovi in Greece. Bull World Health Organ, 1953; 8: 497-511.

10) Cohen JM, Smith DL, Cotter C, et al: Malaria resurgence: a systematic review and assessment of its causes. Malar J. 2012; 11: 122 . 